\title{
OFDM Demodulation in Underwater Time-Reversed Shortened Channels
}

\author{
João Gomes \\ Instituto Superior Técnico \\ Instituto de Sistemas e Robótica \\ Av. Rovisco Pais, 1049-001 Lisboa, Portugal \\ jpg@isr.ist.utl.pt
}

\author{
António Silva, Sérgio Jesus \\ SiPLAB - Universidade do Algarve \\ Campus de Gambelas, 8005-139 Faro, Portugal \\ asilva@ualg.pt, sjesus@ualg.pt
}

\begin{abstract}
This work addresses the problem of OFDM transmission in dispersive underwater channels where impulse responses lasting tens of miliseconds cannot be reliably handled by recently proposed methods due to limitations of channel estimation algorithms. The proposed approach relies on passive time reversal for multichannel combining of observed waveforms at an array of sensors prior to OFDM processing, which produces an equivalent channel with a shorter impulse response that can be handled much more easily. A method for tracking the narrowband residual phase variations of the channel after Doppler preprocessing is proposed. This is a variation of an existing technique that can improve the spectral efficiency of OFDM by reducing the need for pilot symbols. This work also examines techniques to handle sparse impulse responses and proposes a channel estimation method where an $l_{1}$ norm is added to the standard least-squares cost function to transparently induce sparseness in the vector of channel coefficients. Algorithms are assessed using data collected during the UAB'07 experiment, which was conducted in Trondheim fjord, Norway, in September 2007. Data were transmitted with bandwidths of 1.5 and $4.5 \mathrm{kHz}$, and recorded at a range of about $800 \mathrm{~m}$ in a 16-hydrophone array. Significant multipath was observed over a period of at least 30 ms.
\end{abstract}

\section{INTRODUCTION}

Single-carrier modulation and equalization-based reception is currently considered to be the standard method to attain bandwidth-efficient transmission in underwater channels [1]. However, recently published experimental results demonstrating practical OFDM communication in underwater channels at rates in the tens of kbit/s [2], [3] have generated much interest not only by the accomplishment itself, but also because OFDM is an enabling technology that can be integrated with MIMO transmission to attain even greater transmission rates through spatial multiplexing [4].

In most of the reported OFDM experiments mentioned above channel impulse responses are quite favorable, lasting for less than $10 \mathrm{~ms}$. However, in many practically relevant underwater propagation environments at ranges up to about 1 $\mathrm{km}$ channel impulse responses may last much longer than this, on the order of $50 \mathrm{~ms}$. This raises some questions regarding the suitability of the approaches mentioned above for generalpurpose modems that must also operate in severely dispersive underwater channels.

This work aims to contribute to a better understanding of the potential of OFDM by examining results from the UAB'07 ex- periment, which was conducted in Trondheim fjord, Norway, in September 2007. Modulated data were transmitted using several formats with bandwidths of 1.5 and $4.5 \mathrm{kHz}$ (data rates of 1-7 kbps), and recorded at a range of about $800 \mathrm{~m}$ in a 16-hydrophone array. Significant multipath was observed over a period of at least $30 \mathrm{~ms}$, which would call for a rather large OFDM prefix/guard interval and many pilot tones for channel estimation, hence drastically reducing the data rate. Passive time reversal [5] is proposed as a computationally inexpensive preprocessing scheme to shorten the effective channel length to less than $10 \mathrm{~ms}$, so that moderate guard intervals $(30 \mathrm{~ms})$ can be used with a conventional OFDM receiver architecture. The same preamble/postamble signals used for packet synchronization and Doppler precompensation are reused as channel probes for passive time reversal, so the latter entails no loss in efficiency.

Prior work on UAB'07 data included a performance comparison of OFDM and conventional QPSK transmission under identical conditions [6]. Best performance was obtained with QPSK and equalization, but this requires selecting $a$ priori an appropriate set of equalizer parameters, which is a somewhat delicate task. By contrast, an OFDM receiver is more robust to the choice of operating parameters, which is a significant practical advantage. The present paper addresses specific issues of the OFDM receiver architecture, namely, it assesses a method for tracking the residual phase variations of the channel after Doppler preprocessing which can potentially reduce the need for pilot symbols used for channel estimation by using tentative decisions as reference symbols. This technique is a variation of the one proposed in [3], [7] in the presence of narrowband Doppler, which manifests itself as a phase drift with similar rates at all frequencies in the signal band. The impact of estimation techniques for sparse channel impulse responses is also addressed, and it is found that simple coefficient truncation performs poorly with the sinclike shortened equivalent impulse responses that are obtained after passive time reversal. An alternative approach is proposed based on a modified cost function to determine the vector of time-domain impulse response coefficients that includes as a regularization term the $l_{1}$ norm of the desired vector, in addition to the conventional least-squares cost. This approach inherently induces sparseness in the channel estimates, and 
is shown to provide a reduction in error rates at the OFDM receiver output.

\section{OFDM MODEL}

The baseband transmitted OFDM signal is the superposition of $K$ orthogonal subcarrier waveforms

$$
x(t)=\sum_{k=0}^{K-1} \sum_{l} a_{k}(l) u_{k}\left(t-l T_{b}\right),
$$

where $a_{k}(l)$ denotes a point from the $k$-th subcarrier complex constellation in the $l$-th OFDM symbol interval, $u_{k}(t)$ is the corresponding pulse shape, and $T_{b}$ is the OFDM symbol duration including any prefix/postfix. The standard choice is for the pulses $u_{k}(t)$ to be derived from a single rectangular prototype of length $T, \Pi(t / T)$, by exponential modulation with frequencies $f_{k}=k / T$,

$$
u_{k}(t)=e^{j \frac{2 \pi k}{T} t} \Pi\left(\frac{t}{T}\right), \quad \Pi(t) \triangleq \begin{cases}1, & 0 \leq t<1 \\ 0, & \text { otherwise }\end{cases}
$$

For narrowband pulses the total signal bandwidth is approximately $W=K / T$. To enable simplified frequency-domain processing based on the FFT/IFFT, a cyclic prefix (CP) is typically prepended to each OFDM symbol. A CP is absent in the model (2), which corresponds to zero-padded (ZP) OFDM with a guard interval of length $T_{g}=T_{b}-T$. As in [2] a $\mathrm{CP}$ will be synthetically emulated at the receiver by overlapadd, i.e., adding to the start of an OFDM symbol the observed waveform in the trailing guard interval. This strategy neglects the advantages of ZP-OFDM regarding channel identifibility, but it does retain the simplicity of FFT-based processing.

Subcarriers are partitioned into $K_{g}$ guard subcarriers, $K_{n}$ null subcarriers, $K_{p}$ pilot carriers, and $K_{a}$ active carriers, such that $K=K_{g}+K_{n}+K_{p}+K_{a}$. Null symbols $a_{k}=0$ are used in guard and null (unmodulated) subcarriers. The former are located at the upper and lower signal band edges to account for the nonzero rolloff of practical front-end bandpass filters, which may induce strong aliasing distortion when the received baseband OFDM signal is sampled at the critical rate $f_{s}=K / T$ for FFT demodulation. Usually $K_{g} \approx K / 10$. Null, or virtual, subcarriers are spread throughout the signal band to aid in residual Doppler estimation [8]. Known pilot symbols $a_{k}$ are inserted in pilot carriers for channel estimation and/or data-driven or semi-blind residual Doppler estimation. They should preferably be spread uniformly over the signal band to reduce the computational complexity and appropriately sample the channel frequency response. Finally, regular information symbols are inserted in active carriers. Symbol streams for different subcarriers may be generated independently, but more often a single input bit stream is coded, serial-to-parallel converted, and the bit substreams are then mapped into complex symbols. The outer code provides protection against deep channel fades that may affect some of the subcarriers.

\section{Channel Model}

The baseband channel impulse response between the transmitter and the $m$-th receiver hydrophone is denoted by $g_{m}(t)$. This is a static multipath channel where each path is characterized by a complex gain $g_{i}$ and a propagation delay $\tau_{i}$. The emitter and receiver array move with constant relative speed, which induces time scaling of observed waveforms due to Doppler. Specifically, a passband transmission $\tilde{x}(t)=$ $\operatorname{Re}\left\{x(t) e^{j \omega_{c} t}\right\}$ is perceived as

$$
\tilde{x}((1+\beta) t)=\operatorname{Re}\left\{x((1+\beta) t) e^{j \omega_{c} \beta t} e^{j \omega_{c} t}\right\}
$$

when observed over a single path at the receiver, where $\beta$ is the time scaling factor. This effect can be assimilated into the baseband channel model by considering that, over a sufficiently small interval, the channel impulse response becomes time variant such that path delay $\tau_{i}$ changes with time at a constant rate and the phase of path gain $g_{i}$ also evolves linearly [5].

As pointed out in [3], [9] this is a broadband Doppler distortion that affects the various OFDM carriers differently, which is quite different from the narrowband Doppler shifts that are present in wireless radio communications. Assuming that all received paths in all hydrophones experience the same Doppler scaling - a reasonable hypothesis for predominantly horizontal motion - then this broadband distortion may be converted into a narrowband Doppler shift by resampling the received signals, as described in Sec. IV-A.

\section{Demodulation Algorithms}

This section describes the various processing steps that are used to obtain symbol and bit estimates. Broadband Doppler compensation and multichannel combining by passive time reversal are general-purpose techniques that can be used with any type of modulated signal, whereas the ensuing steps are specific to OFDM.

\section{A. Broadband Doppler Compensation}

Let $y_{m}(t)$ denote a baseband Doppler-distorted OFDM signal at the $m$-th hydrophone with scaling factor $\beta$. A resampled compensated signal is generated from $y_{m}(t)$ using an estimate $\hat{\beta}$ as

$$
y_{m}^{\prime}(t)=y_{m}\left(\frac{t}{1+\hat{\beta}}\right) e^{-j \omega_{c} \frac{\hat{\beta} t}{1+\hat{\beta}}} .
$$

As shown in [9], if $\hat{\beta} \approx \beta$ such that $(1+\beta) /(1+\hat{\beta})$ is very close to one, then $y_{m}^{\prime}(t)$ is only affected by a narrowband Doppler shift. It can be expressed as the product of a (multipath-distorted) OFDM signal by a complex exponential $e^{j 2 \pi \epsilon t}$, where

$$
\epsilon=\frac{\beta-\hat{\beta}}{1+\hat{\beta}} f_{c},
$$

and $f_{c}$ is the carrier frequency. The time scaling factor $\beta$ can be estimated in practice by comparing the expected and actual durations of a received packet containing a block of OFDM symbols (or any other type of waveform) [10]. Accurate measurements of packet duration are obtained by scanning for 
prolog/epilog markers (LFM sweeps in this work) that flank each packet. These markers have sufficiently good crosscorrelation properties to be used for frame synchronization as well.

Estimated scaling factors are averaged across sensors, and a single value $\hat{\beta}$ is used to resample all signals.

\section{B. Multichannel Combinining by Passive Time Reversal}

In [3], [9] one FFT demodulator is associated with each of the receiver sensors, and given the observations and channel estimates for a given subcarrier the corresponding symbol is then obtained as the solution of a least-squares problem. This approach is based on the assumption that relatively reliable estimates are available in at least some of the sensors. This hypothesis holds in most of the experimental results presented in [3], [9], where multipath spreads of only a few miliseconds in individual channel impulse responses are much smaller than OFDM symbol lengths and guard intervals. Considerably performance degradation is observed in scenarios that challenge these assumptions, e.g., in a strongly reverberant harbor experiment [9] or at short ranges in shallow water (say, about $500 \mathrm{~m}$ ) where several multiply-reflected paths still contribute significantly to the channel response. Several equalization and channel shortening approaches are proposed in the technical literature on OFDM to overcome the problem of long impulse responses, but one may argue that they detract from the appealing simplicity of the plain FFT-based technique.

Passive time reversal may be viewed as a multichannel combining strategy where measured waveforms directly provide the parameters needed for intersymbol interference mitigation at the receiver [5]. The basic technique is computationally simple and can be applied to any type of modulation. It relies on an impulse-like channel probe that is sequentially sent from that same location where the message $x(t)$ originates, and received at an array (passive time-reversal mirror) with $M$ transducers. The distorted probes are time-reversed and conjugated ${ }^{1}$, convolved with the distorted messages, and summed across sensors to generate a single signal with mild intersymbol interference. In particular, the residual channel impulse response is expected to be sufficiently short to enable OFDM processing regardless of the complexity of the original ocean acoustic channel. Formally, the passive mirror operates on the following received probes $h_{m}(t)$ and messages $y_{m}(t)$

$$
\begin{aligned}
h_{m}(t) & =r(t) * g_{m}(t), \quad r(t) \triangleq p(t) * p^{*}(-t), \\
y_{m}(t) & =x(t) * g_{m}(t) .
\end{aligned}
$$

The assumptions regarding channel impulse responses $g_{m}(t)$ were described in Sec. III. The definition of distorted probes $h_{m}(t)$ in (6) already accounts for pulse compression at the receiver by crosscorrelating with the (time-reversed) transmitted shape $p^{*}(-t)$. In particular, the LFM sweeps used for Doppler compensation and frame synchronization in Sec. IV-A have an impulse-like crosscorrelation and can be reused as

\footnotetext{
${ }^{1}$ In terms of baseband signals time reversal also requires conjugation of channel probes.
}

suitable probes for time reversal, so the proposed multichannel combining approach has no impact on bandwidth efficiency.

The output, $z(t)$, of a passive mirror is generated as

$$
\begin{aligned}
& z(t)=\sum_{m=1}^{M} h_{m}^{*}(-t) * y_{m}(t)=r(t) * \gamma(t) * x(t), \\
& \gamma(t) \triangleq \sum_{m=1}^{M} g_{m}^{*}(-t) * g_{m}(t) .
\end{aligned}
$$

The time reversal property ensures that the sum of medium autocorrelation functions, $\gamma(t)$, is nearly constant over the signal bandwidth for a sufficiently long and dense mirror, so it behaves as an impulse under convolution with $x(t)$. The same holds for the compressed transmitted probe $r(t)$, hence $z(t)$ will be proportional to $x(t)$ and multipath will be eliminated. A practical mirror may not completely cancel the multipath distortion, but as discussed above it need only mitigate it to a point where OFDM demodulation becomes feasible.

It was shown in [5] that resampling for Doppler compensation as described in Sec. IV-A has a negligible impact on passive time reversal under realistic conditions as long as both the distorted probes and packets are identically preprocessed according to (4). Multichannel combining through (8) is therefore carried out on resampled signals without any modification.

\section{Residual Frequency Acquisition}

The presence of a narrowband Doppler shift in the mirror output after applying the broadband compensation technique of Sec. IV-A creates residual intercarrier interference (ICI) and degrades the output SNR. Several methods exist to estimate this residual frequency exploiting the presence of known tones or the redundancy of cyclic prefixes [8]. This work adopts the blind technique based on null subcarriers (commonly known as virtual subcarriers, or VSCs) that was used in [9]. In this method a number of carriers with null information content are spread throughout the signal bandwidth, and a grid search over tentative frequencies is carried out to find the one that minimizes the spillover of energy into those subcarriers in the signal

$$
z^{\prime}(t)=e^{-j 2 \pi \hat{\epsilon} t} z(t) .
$$

Specifically, the Fourier transform of (10) after ZP processing is computed at the $K_{n}$ null subcarrier frequencies and the cost function is evaluated as the squared sum of those coefficients.

In [9] the VSC method is applied independently to each OFDM symbol, but the estimates of $\epsilon$ tend to be somewhat inconsistent for low values of $K$, where few null tones are available (as few as $K_{n}=3$ tones for $K=64$ in the UAB'07 experiment). Because lower values of $K$ also mean shorter OFDM symbols for constant bandwidth, one may then increase the effective number of VSCs by estimating $\epsilon$ based on a sliding window that includes one or two OFDM symbols adjacent to the current one. An alternative blind method is proposed in [11] for constant modulus constellations such as QPSK. It adds to the VSC cost function a term that penalizes the deviation of symbols in active/pilot subcarriers 
from constant modulus, assuming that the duration of the channel impulse response is smaller than $K$. Deriving ICI information from these additional carriers can lead to more accurate estimates of $\epsilon$, but the actual improvements observed in UAB'07 data were modest.

\section{Channel Estimation}

After residual frequency acquisition the compensated mirror output in each OFDM symbol interval $n$ is reduced to a vector of $K$ subcarrier samples $Z_{k}(n), 0 \leq k \leq K-1$ by overlapadding the $\mathrm{ZP}$ and performing Fourier analysis.

Frame synchronization typically aligns a packet in time such that the strongest path in the underlying channel impulse response occurs at delay zero. In underwater communications this is not necessarily the first arrival, which means that the impulse response may have noncausal components that should be accounted for in channel estimation algorithms. This issue is particularly relevant when time reversal is used, as the teoretical channel impulse response associated with $z(t)$ in (8), $h(t) \triangleq r(t) * \gamma(t)$, is a convolution of probe and medium autocorrelation functions, and therefore will be nearly symmetric around delay zero. It will then be assumed that the overall discrete-time impulse response, denoted by $h(n)$, has nonzero support only for $n_{-} \leq n \leq n_{+}$, with $n_{-} \leq 0$, $n_{+} \geq 0$. The DFT coefficients are given by

$$
H_{k}=\sum_{n=n_{-}}^{n_{+}} h(n) e^{-j \frac{2 \pi k}{K} n}, \quad 0 \leq k \leq K-1 .
$$

Let $k_{1}, \ldots k_{C}$ denote a set of carrier indices whose symbols are known. Then the impulse response parameters may be obtained by a least-squares fit

$$
\hat{\mathbf{h}}=\arg \min _{\mathbf{h}}\|\mathbf{b}-\mathbf{A} \mathbf{h}\|_{2}^{2},
$$

where

$$
\mathbf{b}=\left[\begin{array}{c}
Z_{k_{1}} / a_{k_{1}} \\
\vdots \\
Z_{k_{C}} / a_{k_{C}}
\end{array}\right], \quad \mathbf{h}=\left[\begin{array}{c}
h\left(n_{-}\right) \\
\vdots \\
h\left(n_{+}\right)
\end{array}\right]
$$

and $\mathbf{A}$ is the subset of elements from the $K \times K$ DFT matrix in lines $k_{1}, \ldots k_{C}$ and columns $n_{-}, \ldots n_{+}$(modulo $K$ ).

1) Channel and Phase Tracking: In [9] (12) is solved for the set of pilot carriers, which places an upper bound on the dimension of $\mathbf{h}$ as it cannot exceed $K_{p}$ for $\mathbf{A}$ to have full column rank. An alternative approach based on [3] may be envisaged where tentative decisions are used to expand the set of equations and provide more accurate channel estimates. Actually, [3] proposes using pilot tones during the initial symbols of an OFDM packet to obtain a channel estimate $\mathbf{h}$ that can support reasonably reliable tentative decisions, and then switching to pure decision-directed mode and eliminate pilot tones altogether to improve the bandwidth efficiency. This approach rests on the assumption that $\mathbf{h}$ varies slowly over an OFDM packet, so that it can be recursively updated in time using, e.g., exponential weighting

$$
\mathbf{h}(n+1)=\lambda \mathbf{h}(n)+(1-\lambda) \hat{\mathbf{h}}(n), \quad 0 \leq \lambda \leq 1,
$$

where $\hat{\mathbf{h}}(n)$ denotes the estimate derived from the $n$-th OFDM symbol alone. For this technique to succeed residual phase variations between OFDM symbols should be explicitly tracked and their influence eliminated from $\hat{\mathbf{h}}$ so that this vector remains reasonably stable over time. In [3] residual phase variations were modeled as nonuniform Doppler shifts over the subcarriers. An analysis of UAB'07 data after broadband Doppler compensation and residual frequency acquisition found no evidence of such frequency dependent behavior. There were systematic variations in the phase of the estimated frequency-domain coefficients $H_{k}$ as a function of time, but the trend was similar over all subcarriers irrespective of frequency. In light of this, we propose to track a single phase $\theta$, modeled as random walk, and the channel coefficient vector using the following algorithm. From the observed DFT values build the symbol estimates

$$
\hat{a}_{k}(n)=\frac{Z_{k}(n)}{H_{k}(n-1)} e^{-j \theta(n-1)} .
$$

Formulate symbol decisions $\tilde{a}_{k}$ from $\hat{a}_{k}(n) e^{-j \Delta \theta(n-1)}$ in active carriers, where $\Delta \theta(n-1)$ denotes the increment that was added to $\theta(n-2)$ to obtain $\theta(n-1)$, and use the known symbols $a_{k}$ in pilot carriers. This assumes that the phase evolution will have a constant rate over short intervals, so the increment for updating $\theta(n-1) \rightarrow \theta(n)$ should approximately equal $\Delta \theta(n-1)$. Measure the angular discrepancy and average over subcarriers

$$
\Phi_{k}(n)=\operatorname{Im}\left\{\hat{a}_{k}(n) \tilde{a}_{k}^{*}(n)\right\}, \quad \Phi(n)=\frac{1}{C} \sum_{i} \Phi_{k_{i}}(n) .
$$

Filter $\Phi(n)$ through a loop filter $\mathcal{L}$ to obtain a phase increment

$$
\theta(n)=\theta(n-1)+\Delta \theta(n), \quad \Delta \theta(n)=\mathcal{L}\{\Phi(n)\} .
$$

Counter-rotate the observations with the updated phase

$$
Z_{k}^{\prime}(n)=Z_{k}(n) e^{-j \theta(n)},
$$

and use the decisions $\tilde{a}(n)$, or updated ones for the new phase $\theta(n)$, to solve for $\hat{\mathbf{h}}(n)$ through (12) or a similar criterion. Update the smoothed channel estimate using (14) and calculate its DFT. Repeat (15) with updated $H_{k}(n), \theta(n)$ to obtain final symbol estimates. As in [3], tentative decisions on active carriers are best ommitted from (16) until phase estimates can be relied upon.

2) Alternative Channel Estimation Methods: Having an estimate of the time-domain impulse response vector $\mathbf{h}$ it becomes possible to take advantage of the fact that energy is often concentrated around a few arrivals, and low-value coefficients can be set to zero. This truncation approach is termed channel sparsing in [7]. In addition to potentially reducing the computational complexity of receiver algorithms, sparsing may actually improve the receiver performance by eliminating jitter in small coefficients that do not meaningfully model any channel characteristics.

The overall channel impulse response after passive time reversal is typically narrow and sinc-like, so the above argument should hold and dropping small coefficients could 
prove beneficial as well. The truncation approach of [7] is very simple, as it sets to zero any coefficent whose magnitude is smaller than a given fraction (e.g., 20\%) of the strongest coefficient. The present work examines an alternative method inspired by recent developments in compressed sensing which have shown that even a severely underdetermined system of equations $\mathbf{A} \mathbf{h}=\mathbf{b}$ with sparse $\mathbf{h}$ can still be solved if the $l_{1}$ norm of $\mathbf{h}$ is adopted as a regularization term. Specifically, using the formulation

$$
\hat{\mathbf{h}}=\arg \min _{\mathbf{h}}\|\mathbf{b}-\mathbf{A} \mathbf{h}\|_{2}^{2}+\alpha\|\mathbf{h}\|_{1}
$$

examples have been shown where $\mathbf{h}$ is recovered even when the number of equations is only $10 \%$ of the number of unknowns in that vector (most of which are actually zero, albeit in unknown positions) [12]. The key to this remarkable behavior is the fact that the $l_{1}$ norm can act as a good surrogate for the cardinality of a vector $\left(l_{0}\right.$ norm), i.e., the number of nonzero components. Adopting cardinality as a penalization term therefore tends to produce a solution where many coefficients are exactly zero. Using $l_{1}$ norm regularization the approximation problem can be formulated in convex form and solved using efficient numerical algorithms [13], a crucial advantage over the $l_{0}$ norm.

In the present case the fitting problem is not underdetermined, but the formulation (19) is still expected to encourage the annihilation or attenuation in $\mathbf{h}$ of small coefficients. This is perhaps more clearly understood from the remark in [12] that (19) is closely related to the problem

$$
\min _{\mathbf{h}}\|\mathbf{h}\|_{1} \text { subject to }\|\mathbf{b}-\mathbf{A} \mathbf{h}\|_{2}^{2} \leq \delta,
$$

which explicitly minimizes the "cardinality" of $\mathbf{h}$ subject to an accuracy requirement on the residual vector.

In this work the problem (19) is solved using a generalpurpose convex optimization package, but for this particular cost function the GPSR algorithm [12] can be used in practice with lower computational complexity. This algorithm can handle very large scale problems, making it quite suitable for channel estimation in OFDM even with large numbers of carriers and pilot symbols.

\section{EXPERIMENTAL RESULTS}

The receiver was tested using experimental data collected by the University of Algarve during the UAB'07 experiment, which was conducted in Trondheim fjord, Norway, in September 2007. The transmitter was suspended from a fixed platform $10 \mathrm{~m}$ from shore, at a depth of about $5 \mathrm{~m}$. The receiver was a vertical array with 16 uniformly-spaced hydrophones from $6 \mathrm{~m}$ to $66 \mathrm{~m}$ depth, suspended from a drifting Acoustic Oceanographic Buoy (AOB) developed by the University of Algarve. The communication range was approximately $800 \mathrm{~m}$, the bottom depth gradually increasing from $10 \mathrm{~m}$ at the transmitter to about $100 \mathrm{~m}$ at the receiver location. Signals were transmitted around a carrier frequency of $5.5 \mathrm{kHz}$ with bandwidths of $1.5 \mathrm{kHz}$ or $4.5 \mathrm{kHz}$ and variable number of subcarriers. Tab. I summarizes the parameters for

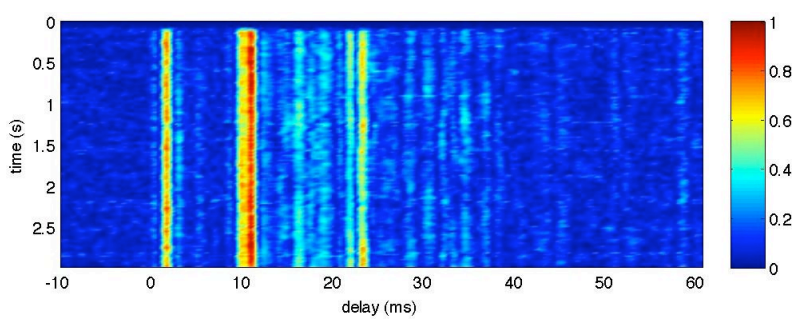

(a)

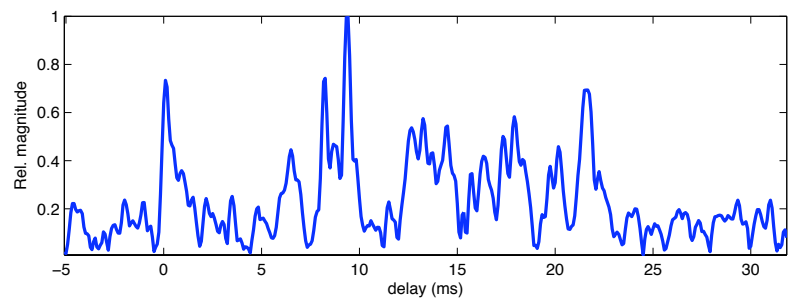

(b)

Fig. 1: Amplitude-normalized estimated channel response at depth $22 \mathrm{~m}$ (hydrophone \#5). Doppler precompensation was performed according to Sec. IV-A (a) Evolution over a $3 \mathrm{~s}$ packet (b) Snapshot

six different types of OFDM packets denoted by O1-6. A total of $K_{g} \approx 0.15 K$ guard carriers were reserved at the band edges for practical front-end filtering by a raised-cosine filter. As discussed in Secs. IV-A and IV-B, packets were flanked by start/stop LFMs, each sweeping across $5.5 \pm 2.25 \mathrm{kHz}$ in 20 $\mathrm{ms}$, followed (or preceded) by a $50 \mathrm{~ms}$ silent period.

Packet contents are generated from a single bit stream that is convolutionally encoded with a rate $1 / 2$ code (constraint length 7 , octal generators $(133,171)$ [14]), punctured by eliminating 1 out of 3 bits to increase the rate to $3 / 4$. The coded bits are then randomly interleaved and partitioned into subblocks to be mapped into OFDM symbols as needed by the various packet formats. Decoding at the receiver uses the Viterbi algorithm with traceback length $5 \times 7$ operating on hard symbol decisions.

Unless otherwise noted, in the experimental results reported below one out of four subcarriers are used as pilots.

a) Channel response: Fig. 1 shows the evolution of the estimated $^{2}$ channel impulse response at depth $22 \mathrm{~m}$ over a period of $3 \mathrm{~s}$, after Doppler precompensation as described in Sec. IV-A. The main arrivals are relatively stable, and the multipath spread is about $30 \mathrm{~ms}$, although low-amplitude arrivals can be detected at delays of up to $100 \mathrm{~ms}$ (not shown). These results show that the multipath distortion is more severe than in other reported OFDM experiments [3], [9].

b) MRC versus Time-Reversed OFDM: Tab. II compares symbol error rates (SER) and coded bit error rates (BER) of time-reversed OFDM (TR-OFDM) and the multichannel combining approach of [3], [9] (MRC-OFDM), where one FFT demodulator is used for each receiver sensor and channel

\footnotetext{
${ }^{2}$ The channel was estimated by recursive identification from a single-carrier packet. More details can be found in [6].
} 
TABLE I: OFDM signal parameters in the UAB'07 experiment

\begin{tabular}{|c|c|c|c|c|c|c|}
\hline \hline Packet type & O1 & O2 & O3 & O4 & O5 & O6 \\
\hline \hline Bandwidth $[\mathrm{kHz}]$ & 1.5 & 1.5 & 1.5 & 4.5 & 4.5 & 4.5 \\
\hline Subcarriers $K$ & 64 & 128 & 256 & 128 & 256 & 512 \\
\hline Carrier spacing $[\mathrm{Hz}]$ & 23.4 & 11.7 & 5.8 & 35.1 & 17.6 & 8.8 \\
\hline Active interval $T[\mathrm{~ms}]$ & 42.7 & 85.3 & 170.7 & 28.4 & 56.9 & 113.8 \\
\hline Guard interval $T_{g}[\mathrm{~ms}]$ & \multicolumn{7}{|c|}{30} & \\
\hline Null carriers $K_{n}$ & 3 & 5 & 10 & 5 & 10 & 20 \\
\hline Guard carriers $K_{g}$ & 10 & 20 & 40 & 20 & 40 & 78 \\
\hline Number of symbols $N$ & 40 & 30 & 15 & 60 & 40 & 20 \\
\hline \hline Constellation & \multicolumn{7}{|c|}{ QPSK } \\
\hline \hline
\end{tabular}

TABLE II: Performance of MRC-OFDM and TR-OFDM using 1 out of 4 pilot subcarriers

\begin{tabular}{|c|c|c|c|c|c|c|}
\hline \hline PKT & O1 & O2 & O3 & O4 & O5 & O6 \\
\hline \hline \multicolumn{7}{|c|}{ MRC-OFDM } \\
\hline SER & 0.52 & 0.11 & $6.1 \mathrm{e}-3$ & 0.45 & 0.39 & 0.06 \\
\hline BER & 0.79 & 0.32 & 0 & 0.77 & 0.78 & 0.12 \\
\hline \hline \multicolumn{7}{|c|}{ TR-OFDM } \\
\hline SER & 0.05 & 0.01 & $3.5 \mathrm{e}-3$ & 0.08 & 0.05 & $3.4 \mathrm{e}-3$ \\
\hline BER & 0.10 & $9 \mathrm{e}-4$ & $9 \mathrm{e}-4$ & 0.23 & 0.08 & 0 \\
\hline
\end{tabular}

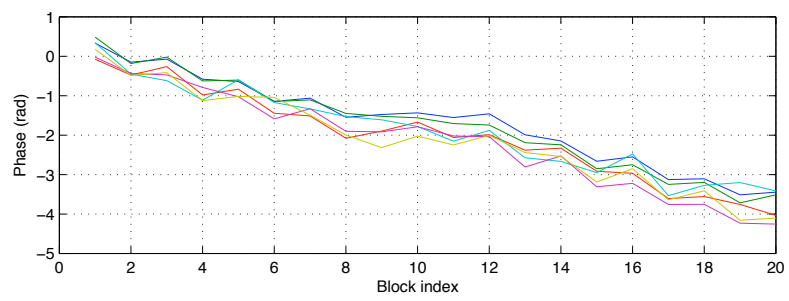

Fig. 2: Evolution of phase in the estimated channel frequency response for an $\mathrm{O} 6$ packet $(4.5 \mathrm{kHz}$ bandwidth, 512 subcarriers)

estimates are combined to yield more reliable symbol decisions. OFDM symbols are processed independently, as in [9]. MRC-OFDM yields poor results in the UAB'07 data set because the number of pilot symbols $\left(K_{p}=K / 4\right)$ does not provide enough degrees of freedom to properly model the relatively long channel response in each sensor. By contrast, time reversal compresses the equivalent impulse response to less than $10 \mathrm{~ms}$, which is sufficiently short for acceptable channel modeling. For the level of obtained SERs a more powerful outer code such as the one used in [7] would have significantly improved the bit error rates.

c) Channel and Phase Tracking: Fig. 2 shows the evolution of the phase of the estimated frequency response as a function of time for several subcarrier frequencies in a $\mathrm{O} 6$ packet $(4.5 \mathrm{kHz}$ bandwidth, 512 subcarriers). The slope is very similar for all frequencies and deviates significantly from the model proposed in [3] assuming broadband Doppler distortion. Fig. 3 shows some of the internal variables and performance metrics for demodulation of that packet using the algorithm of Sec. IV-D1. In this case the loop filter $\mathcal{L}$ in (17) is just a unit gain, and the forgetting factor used for channel tracking

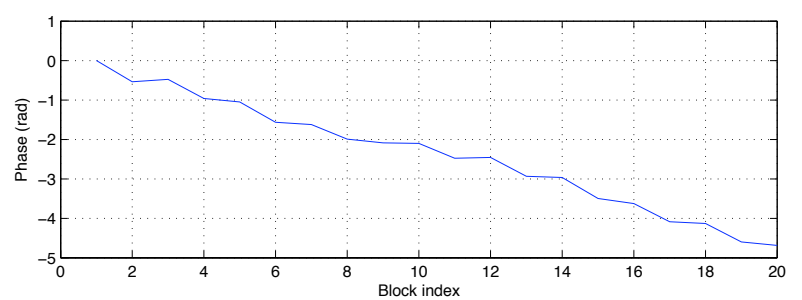

(a)

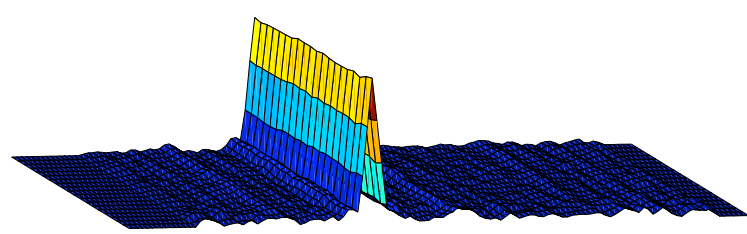

(b)

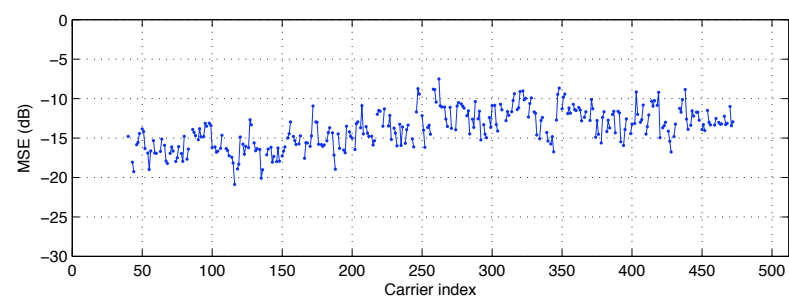

(c)

Fig. 3: Demodulation of an O6 packet using channel and phase tracking (a) Evolution of estimated phase (b) Evolution of estimated channel impulse response (c) MSE over active carriers

through (14) is $\lambda=0.1$. Tab. III provides symbol and bit error rates for this algorithm and the one proposed in [3]. Tracking is based on pilot tones alone during the first 5 OFDM symbols, after which symbol decisions on the remaining active subcarriers are taken into account to improve the estimation accuracy. These results show similar performance by both algorithms, but no clear advantage in terms of error rates is observed over the algorithm of Tab. II that processes OFDM symbols independently. However, note that in this example pilot symbols are still present after switching to decisiondirected mode, as the raw symbol decisions that were used 
TABLE III: Performance of OFDM receivers with channel/phase tracking

\begin{tabular}{|c|c|c|c|c|c|c|}
\hline \hline PKT & O1 & O2 & O3 & O4 & O5 & O6 \\
\hline \hline \multicolumn{7}{|c|}{ Broadband phase model [3] } \\
\hline SER & 0.04 & 0.12 & $1.3 \mathrm{e}-3$ & 0.12 & 0.04 & $3 \mathrm{e}-4$ \\
\hline BER & 0.06 & 0.26 & 0 & 0.31 & 0.04 & 0 \\
\hline \hline \multicolumn{7}{|c|}{ Narrowband phase model } \\
\hline SER & 0.05 & 0.03 & $3.5 \mathrm{e}-3$ & 0.12 & 0.03 & $5 \mathrm{e}-4$ \\
\hline BER & 0.08 & 0.04 & $1.2 \mathrm{e}-3$ & 0.32 & 0.02 & 0 \\
\hline \hline
\end{tabular}

TABLE IV: Impact of mixed $l_{1}, l_{2}$ impulse response estimation

\begin{tabular}{|c|c|c|c|c|c|c|}
\hline \hline PKT & O1 & O2 & O3 & O4 & O5 & O6 \\
\hline \hline \multicolumn{7}{|c|}{ TR-OFDM } \\
\hline SER & $4.6 \mathrm{e}-3$ & $1.3 \mathrm{e}-3$ & $1.7 \mathrm{e}-3$ & 0.06 & 0.05 & 0.02 \\
\hline BER & $1.8 \mathrm{e}-3$ & 0 & $2 \mathrm{e}-3$ & 0.10 & 0.05 & 0.01 \\
\hline \hline \multicolumn{7}{|c|}{ Narrowband phase model } \\
\hline SER & $1.3 \mathrm{e}-3$ & 0.02 & $2.6 \mathrm{e}-3$ & 0.10 & 0.06 & $4.2 \mathrm{e}-3$ \\
\hline BER & 0 & 0.03 & $3 \mathrm{e}-4$ & 0.23 & 0.10 & $1.9 \mathrm{e}-3$ \\
\hline \hline
\end{tabular}

to track the phase are not reliable enough to attain convergence in all packets. Integrating the outer decoder into the symbol decision process would have increased its reliability, allowing some or all of the pilot tones to be freed for data transmission and hence increase the spectral efficiency when using pahse/channel tracking algorithms.

In this case, the frequency-dependent phase model of [3] does not match the observations well, but channel tracking is sufficiently fast so that incorrect prediction of the phase at upper frequencies is transparently compensated by counterrotation of the channel coefficients $H_{k}$ at those frequencies. If a larger value of the channel tracking constant $\lambda$ had been used then these phase discrepancies would lead to a degradation of the smoothed channel estimates.

d) Alternative Channel Estimation: In contrast to the significant performance improvements shown in [7], sparsing by coefficient truncation of the overall impulse response after time reversal yielded poor results. Apparently, modeling of this type of sinc-like response requires low-value coefficients to attain proper interpolation in the frequency domain. Much better results were attained using the mixed $l_{1}, l_{2}$ cost function (19) with $\alpha=1.1$. This empirical value of $\alpha$ seems to be a good compromise for all packets. Tab. IV lists symbol and bit error rates for the algorithm that processes symbols independently and for the narrowband phase and channel tracking receiver. Both algorithms improve upon their counterparts based on least-squares channel estimation at the expense of greater computational complexity. As before, their relative performance remains similar.

\section{CONCLUSION}

This work examined OFDM receiver architectures based on passive time reversal as a multichannel combining preprocessing step for impulse response shortening. This was shown to be more reliable than an alternative approach where multichannel combining is performed after FFT processing and channel estimation at individual sensors, which fails on the UAB' 07 data set due to the long impulse responses that would require a very large proportion of pilot tones for channel estimation. Time reversal also appears to stabilize the equivalent impulse response, reducing the impact of individual channel variations.

A modification of the phase tracking method of [3] was proposed to account for the observed nearly frequencyindependent evolution of the phase of channel coefficients. Its performance was found to be very similar to the original method, but it puts less strain on the channel tracking recursion because channel estimation does not need to compensate for modeling mismatches of the phase evolution. Performance is also similar to independent processing of OFDM symbols, but the tracking method can reduce the number of required pilot symbols when tentative decisions are sufficiently reliable.

Issues of sparse channel estimation were also addressed. Unexpectedly, the simple coefficient truncation method of [7] failed for reasons that are still not very clear, but appear to be related to the specific sinc-like shape of equivalent timereversed impulse responses, where low-level coefficients are not necessarily neglectable. An alternative approach for sparse channel estimation based on a modified least-squares cost function that includes an additional $l_{1}$ term for regularization was examined and found to provide significant improvements in error rates over plain least-squares estimation in several of the packets. This approach shows some potential, but requires further research to better understand when it can improve upon least-squares estimation. Issues of algorithmic complexity for this method were also absent from this study, but need to be addressed in the future.

\section{ACKNOWLEDGMENT}

This work was supported by Fundação para a Ciência e a Tecnologia through project PTDC/EEA-TEL/71263/2006 and ISR/IST plurianual funding.

\section{REFERENCES}

[1] D. B. Kilfoyle and A. B. Baggeroer, "The state of the art in underwater acoustic telemetry," IEEE Journal of Oceanic Engineering, vol. 25, no. 1, pp. 4-27, January 2000.

[2] B. Li, S. Zhou, M. Stojanovic, and L. Freitag, "Pilot-tone based ZPOFDM demodulation for an underwater acoustic channel," in Proceedings of MTS/IEEE OCEANS'06, Boston, MA, USA, September 2006.

[3] M. Stojanovic, "Low complexity OFDM detector for underwater acoustic channels," in Proceedings of MTS/IEEE OCEANS'06, Boston, MA, USA, September 2006.

[4] B. Li, S. Zhou, M. Stojanovic, L. Freitag, J. Huang, and P. Willett, "MIMO-OFDM over an underwater acoustic channel," in Proceedings of MTS/IEEE OCEANS'07, Vancouver, Canada, September 2007.

[5] J. Gomes, A. Silva, and S. Jesus, "Adaptive spatial combining for passive time-reversed communications," Journal of the Acoustical Society of America, vol. 124, no. 2, pp. 1038-1053, August 2008.

[6] — , "Experimental assessment of time-reversed OFDM underwater communications," in Proceedings of Acoustics'08, Paris, France, June 2008.

[7] M. Stojanovic, "OFDM for underwater acoustic communications: Adaptive synchronization and sparse channel estimation," in Proceedings of the International Conference on Acoustics, Speech and Signal Processing (ICASSP'08), Las Vegas, Nevada, USA, March 2008.

[8] M. Morelli, C.-C. J. Kuo, and M.-O. Pun, "Synchronization techniques for orthogonal frequency division multiple access (OFDMA): A tutorial review," Proceedings of the IEEE, vol. 95, no. 7, pp. 1394-1427, July 2007. 
[9] B. Li, S. Zhou, M. Stojanovic, L. Freitag, and P. Willett, "Multicarrier communication over underwater acoustic channels with nonuniform doppler shifts," To appear in IEEE Journal of Oceanic Engineering, 2008.

[10] B. S. Sharif, J. Neasham, O. R. Hinton, and A. E. Adams, "A computationally efficient doppler compensation system for underwater acoustic communications," IEEE Journal of Oceanic Engineering, vol. 25, no. 1, pp. 52-61, January 2000.

[11] M. Ghogho and A. Swami, "Blind frequency-offset estimator for OFDM systems transmitting constant-modulus symbols," IEEE Communications Letters, vol. 6, no. 8, pp. 343-345, August 2002.

[12] M. A. T. Figueiredo, R. D. Nowak, and S. J. Wright, "Gradient projection for sparse reconstruction: Application to compressed sensing and other inverse problems," IEEE Journal of Selected Topics in Signal Processing, vol. 1, no. 4, pp. 586-597, December 2007.

[13] S. Boyd and L. Vandenberghe, Convex Optimization. Cambridge University Press, 2004.

[14] J. G. Proakis, Digital Communications, 4th ed. McGraw-Hill Book Company, 2000. 